\title{
Fibroid Expulsion after Uterine Artery Embolization
}

\author{
Emily Ochmanek, DO ${ }^{1}$ Matthew A. Brown, MD ${ }^{1}$ Paul J. Rochon, MD, FSIR ${ }^{1}$ \\ ${ }^{1}$ Division of Interventional Radiology, University of Colorado \\ Anschutz Medical Campus, Aurora, Colorado \\ Address for correspondence Paul J. Rochon, MD, FSIR, Department of \\ Radiology, University of Colorado School of Medicine, $12401 \mathrm{E}$. 17th \\ Avenue, Mailstop L954, Aurora, CO 80045 \\ Semin Intervent Radiol 2019;36:126-132 \\ (e-mail: paul.rochon@ucdenver.edu).
}

\author{
Abstract \\ Keywords \\ - fibroid expulsion \\ - uterine artery \\ embolization \\ - deep vein thrombosis \\ - iliac vein stent \\ - interventional \\ radiology
}

Uterine artery embolization (UAE) is a ubiquitous procedure, and a broadly recognized alternative to surgical interventions for symptomatic leiomyomata when uterine preservation is desired. Aside from postembolization syndrome (typically considered an expected feature of recovery), the most frequently described complications are temporary or permanent amenorrhea and lingering vaginal discharge. Less frequently described complications include fibroid expulsion (FE), protracted or refractory pain, infection, urinary retention, and access-related injuries. Reported rates of transcervical FE vary in the literature from 3 to $50 \%$ but are most often quoted to be around 5 to $15 \%$. Certain features predispose a patient to FE, including size and location of the tumor, with pedunculated submucosal, submucosal, and transmural lesions considered to be "high risk." While the optimal management of FE has not been definitively determined, high rates of nonoperative management of FE are noted in the literature. This article describes a case in which a fibroid was expulsed following UAE, as well as the management of the complication. A literature review and recommendations for the management of $\mathrm{FE}$ is also given.
A 38-year-old female was referred for hematology consultation for evaluation of an unprovoked left lower extremity deep vein thrombosis. Given the degree of swelling (left thigh was approximately twice the diameter of the right, with severe pretibial edema in the lower leg) as well as severity of symptoms (pain, impaired ambulation/activity), despite some improvement on anticoagulation with rivaroxaban, the interventional radiology (IR) service was consulted. She also complained of left lower quadrant/pelvic fullness, along with urinary urgency. She reported heavy menstrual bleeding over the past year, and was found to be anemic, with hemoglobin and hematocrit levels of $7.5 \mathrm{~g} / \mathrm{dL}$ and 27.4 , respectively. She was started on iron supplementation. Evaluation for an underlying cause of hypercoagulability was unrevealing.

Computed tomography (CT) of the abdomen and pelvis was obtained at an IR clinic visit the following week, which revealed an enlarged, myomatous uterus with a volume of more than 2,000 mL causing mass effect upon the left common iliac vein, with marked luminal narrowing to $\sim 3 \mathrm{~mm}$. Exten- sive DVT was also present in the expanded left common iliac vein extending into the visualized femoral vein (-Fig. 1).

At her initial consultation, the recommendation was made for thrombolysis/thrombectomy of her left lower extremity DVT to decrease severity of postthrombotic syndrome (PTS), along with potential left common iliac vein stent for likely May-Thurner syndrome based on CT findings, though only after treating the additional vascular compression by her enlarged, myomatous uterus. Options were presented, including uterine artery embolization (UAE), myomectomy, and hysterectomy. Gynecology referral was placed for discussion of surgical options, and magnetic resonance imaging (MRI) ordered for better characterization of the fibroids and her pelvic anatomy. MRI confirmed the presence of a markedly enlarged uterus secondary to the posteriorly located $12.3-\mathrm{cm}$ myometrial leiomyoma (-Fig. 2). The endometrial cavity was displaced anteriorly and to the right but was otherwise unremarkable. Mass effect from the uterus also resulted in mild right hydroureteronephrosis.
Issue Theme Management of Complications in IR; Guest Editor, Robert K. Ryu, MD, FSIR
Copyright $\odot 2019$ by Thieme Medical Publishers, Inc., 333 Seventh Avenue, New York, NY 10001, USA. Tel: +1(212) 584-4662. 

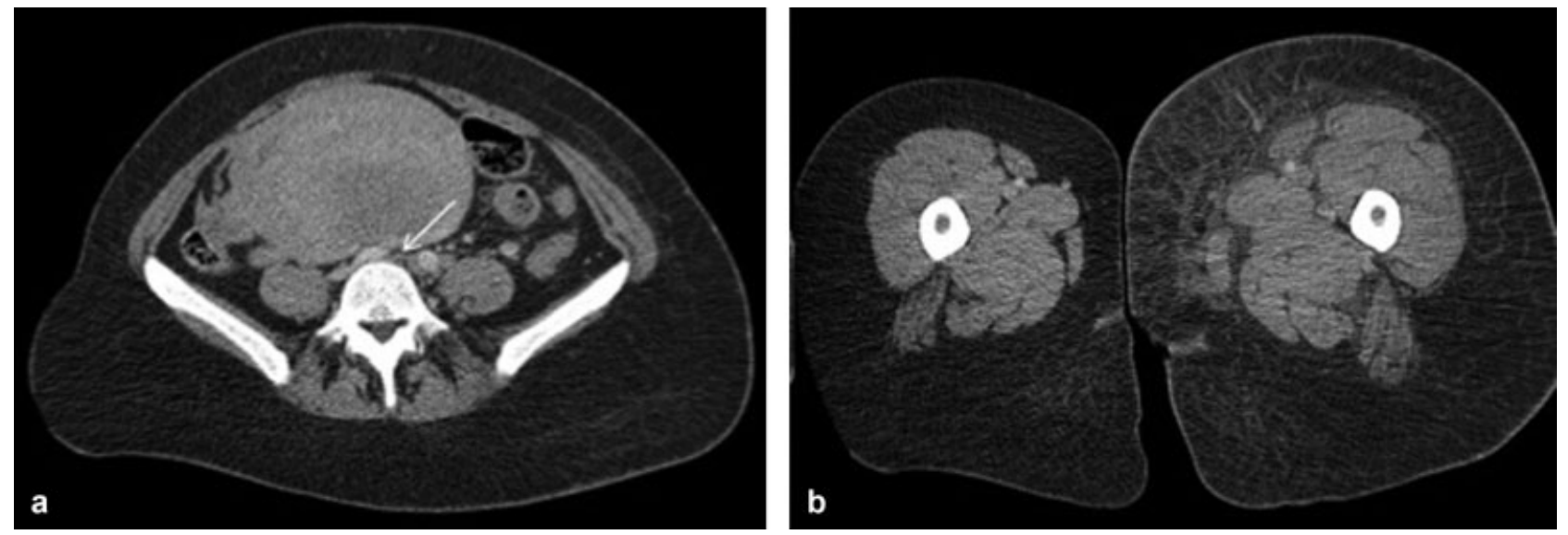

Fig. 1 (a) Postcontrast axial CT images of the pelvis and proximal thighs reveal an enlarged fibroid uterus with a volume of 2,132 $\mathrm{mL}$ causing mass effect upon the left common iliac vein (arrow) with associated marked luminal narrowing. (b) Extensive left lower extremity DVT extending from the left common iliac vein into the external and internal iliac veins, the common femoral, femoral, and visualized profunda femoris veins with asymmetric edema in the left lower extremity.

A more detailed menstrual history was elicited at her gynecology consultation, which revealed that her previously heavy periods had become extreme on rivaroxaban (Janssen Pharmaceuticals, Raritan, NJ), requiring changing 10 pads and large tampons every 20 to 30 minutes for 2 to 3 days. She also had lightheadedness and dizziness related to anemia; her hemoglobin level had dropped to $6.8 \mathrm{~g} / \mathrm{dL}$. The patient stated an interest in fertility preservation. In the assessment of her gynecologist, the patient was felt to be a relatively high-risk surgical candidate in the setting of her extensive DVT and need for perioperative anticoagulation bridging, and thus UAE versus a course of Depo-Lupron to shrink the fibroid, followed by myomectomy, versus hysterectomy in the future was the recommended treatment. The patient opted to pursue UAE, and weekly iron infusions were initiated until her procedure, scheduled approximately 1 month after her initial IR consultation.

\section{Procedure 1}

She underwent an uneventful UAE via a left transradial approach. The left uterine artery was selected first, and embolization performed with nine vials of 700 to $900 \mu \mathrm{m}$ Embospheres (Merit Medical, South Jordan, UT) to five-beat stasis. The right uterine artery was subsequently selected, and three vials of 500 to $700 \mu \mathrm{m}$ Embospheres delivered to five-beat stasis (-Fig. 3). At her 3-week postprocedure clinic visit, she described symptoms typical of postembolization syndrome, including abdominal pain and feeling flushed for approximately 2 weeks, which had since resolved, along with
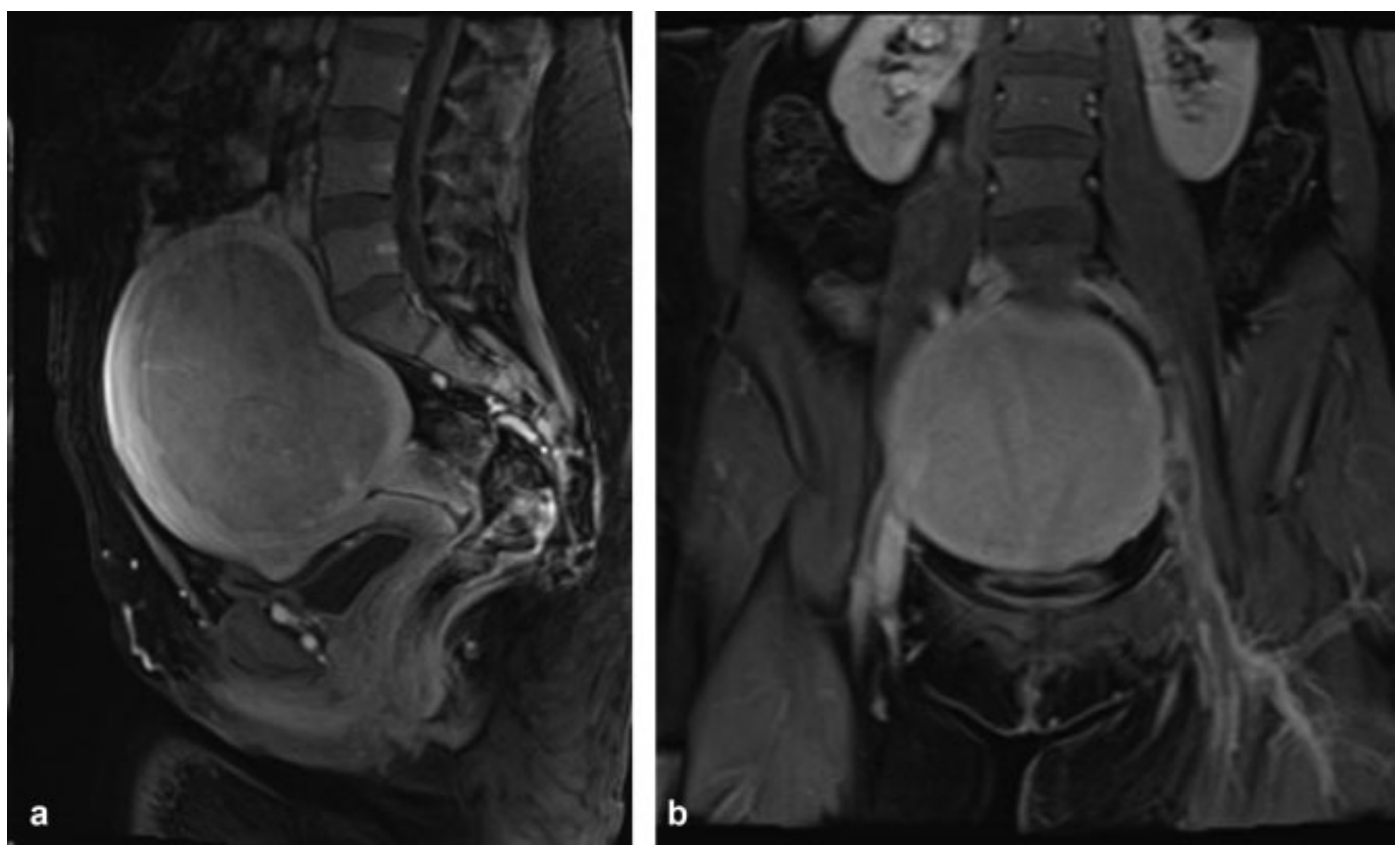

Fig. 2 Sagittal (a) and coronal (b) T1 postcontrast with fat saturation MR images show a markedly enlarged uterus secondary to posterior position myometrial fibroid $(12.3 \mathrm{~cm})$. 

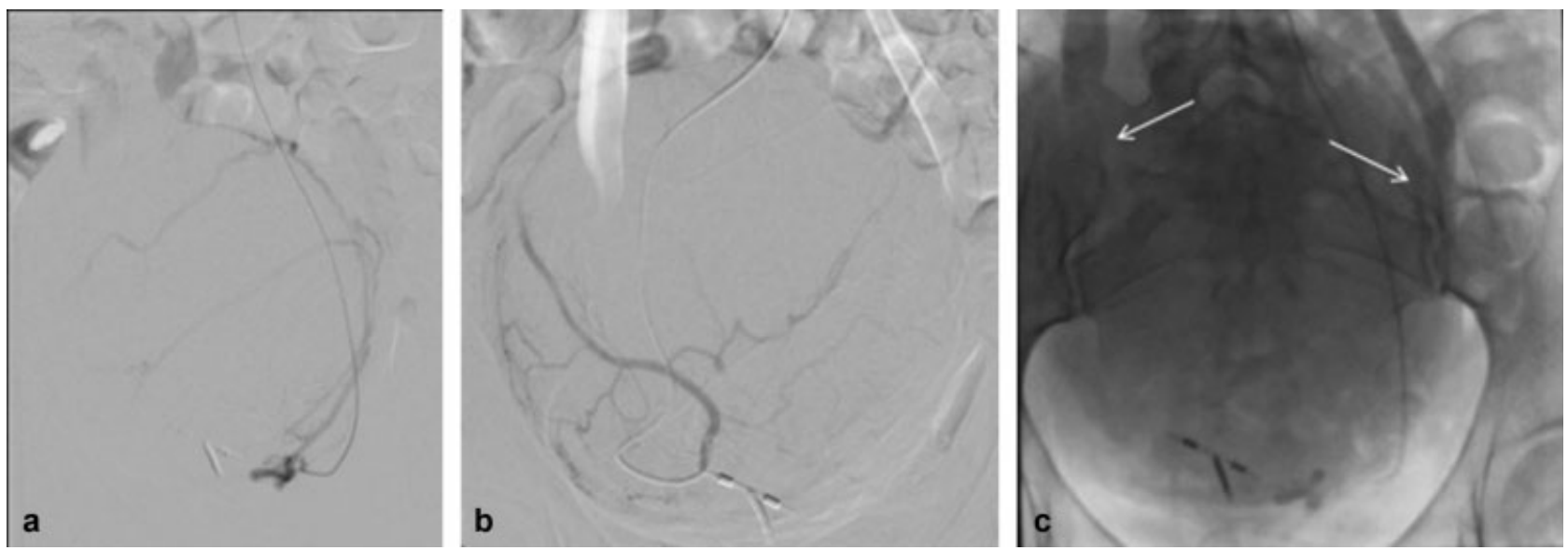

Fig. 3 Digital subtraction angiographic images from UAE, showing selective injections from the hypertrophied left (a) and right (b) uterine arteries. Embolization was performed with nine vials of 700-900 $\mu \mathrm{m}$ Embospheres on the left, followed by three vials of 500 to $700 \mu \mathrm{m}$ Embospheres on the right. Completion fluoroscopic spot image (c) demonstrates hydroureter, right greater than left (arrows), due to extrinsic compression from the massively enlarged, contrast-stained uterus.

some spotting. She had resumed normal activities, including working out with her personal trainer. A 3-month follow-up MRI was ordered to serve as a new baseline prior to her future venous reconstruction.

\section{Complication}

Approximately 4 weeks post-UAE, she complained of increased pelvic pain/cramping, vaginal bleeding, and dizziness that had slowly progressed over several days. She also had flushing, rigors, and sweats, but no fevers or foulsmelling vaginal discharge. Laboratory tests showed no leukocytosis and a normal hemoglobin. Symptoms were felt to be secondary to necrosis of the fibroid, and an urgent MRI was ordered to evaluate for the extent of necrosis.

The MRI revealed complete devascularization of the fibroid, which was slightly decreased in size, though noted to be protruding into the endocervical canal ( - Fig. 4). The possibility of dilation and curettage and/or hysterectomy was discussed with the patient. She was started on prophy- lactic antibiotics, and weekly blood tests and phone calls were arranged.

Approximately 2 weeks later, she presented to the emergency department (ED) with prolapsing of the fibroid. On pelvic examination, a globular, flesh-colored mass was seen protruding from the cervix measuring $\sim 6 \mathrm{~cm}$ in diameter. This mass was nontender, and there was no associated bleeding or erythema. An ultrasound of the left lower extremity to evaluate the status of the deep vein thrombus for procedural and surgical planning was also obtained and demonstrated chronic thrombus from common femoral vein to the common iliac vein ( - Fig. 5). She was discharged from the ED and scheduled for a myomectomy within a week. A Gunther Tulip infrarenal inferior vena cava (IVC) filter (Cook Medical, Bloomington, IN) was placed for perioperative prophylaxis, and her rivaroxaban held for 24 hours preoperatively (-Fig. 6). Exam under anesthesia revealed an 11-week-sized uterus, decreased from 18 -week pre-UAE. Hysteroscopic myomectomy was performed along with dilation and curettage to remove the necrotic fibroid. The procedure was complicated by uterine
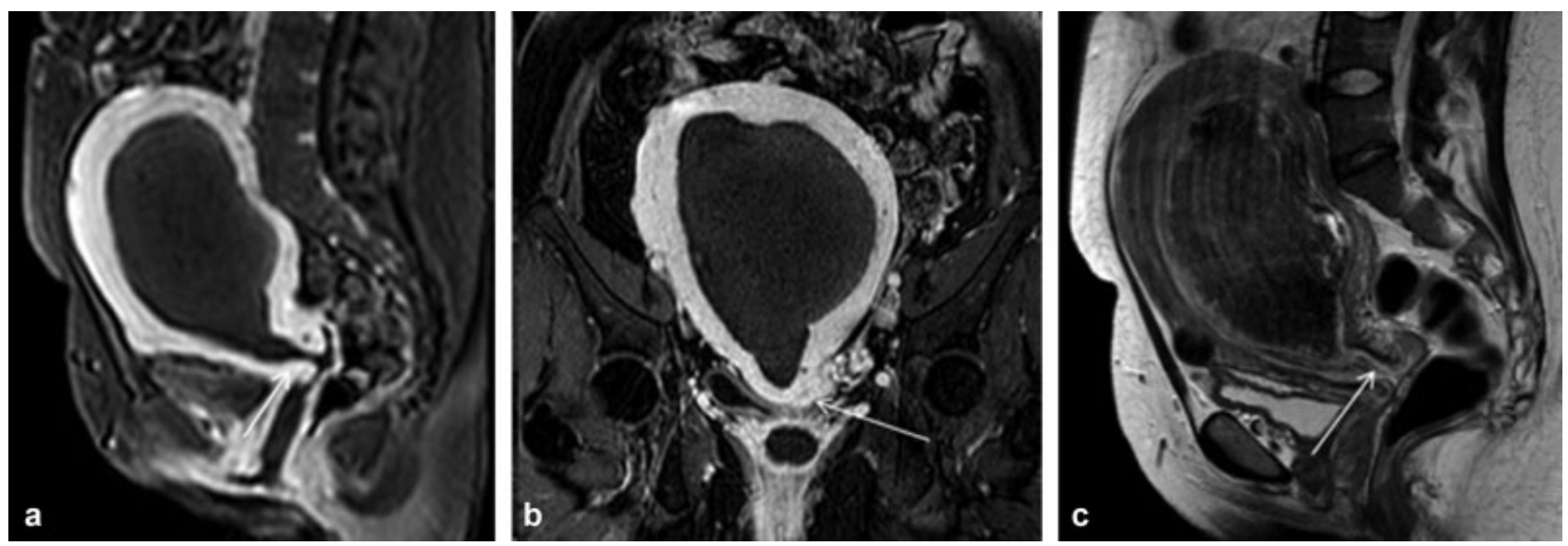

Fig. 4 Sagittal (a) and coronal (b) T1 postcontrast with fat saturation and T2 sagittal (c) MR images post-UAE complete devascularization of the large submucosal uterine fibroid with mild interval decrease in size of the fibroid. The lowermost portion of the embolized fibroid slightly protrudes into the endocervical canal (arrows). 


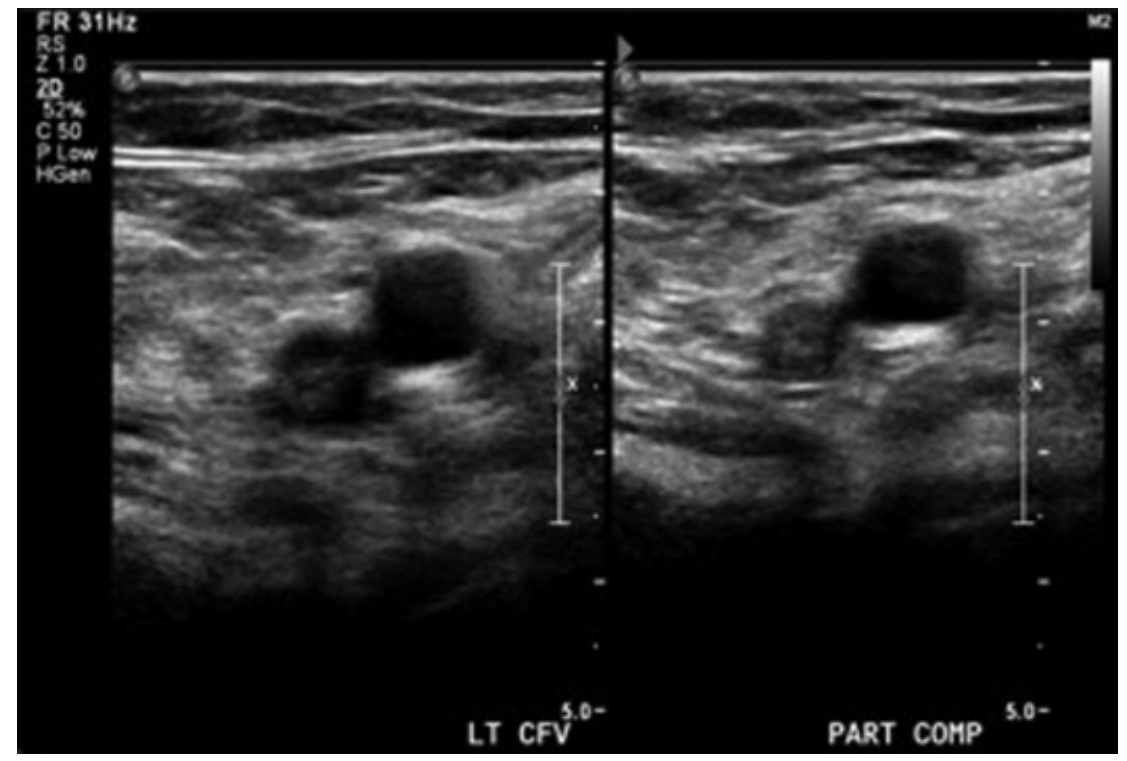

Fig. 5 Grayscale ultrasound images showing chronic appearing nonocclusive deep venous thrombosis in the left partially compressible external iliac vein extending to the left common femoral vein confluence with the great saphenous vein.

bleeding requiring intrauterine Foley balloon placement, but this rapidly resolved, and she was discharged on postoperative day 1 , back on her anticoagulation.

She was evaluated in IR clinic 1 month after surgery. The patient felt well, without further pain, bleeding, or prolapsing tissue. An interval pelvic MRI showed complete treatment response following myomectomy, with a normal-appearing

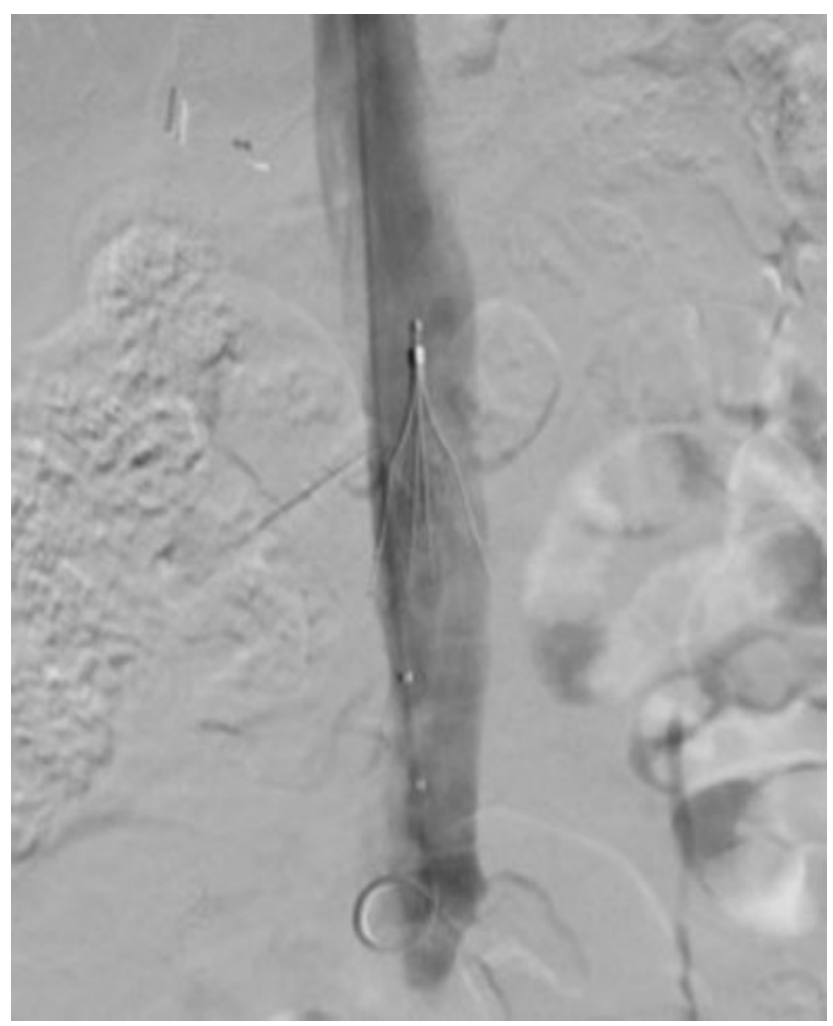

Fig. 6 Digital subtraction angiographic image following placement of a Gunther-Tulip optional filter placed in the infrarenal inferior vena cava for perioperative prophylaxis. uterus (-Fig. 7). Her menstrual flow postoperatively had considerably improved, which she characterized as "light." She remained interested in pursuing venous reconstruction. On physical examination, there was persistent left lower extremity edema, but no erythema or ulceration (C3EsAdPo). A CT venogram was ordered for preprocedural planning. This showed (near) occlusion of the left common iliac vein by the

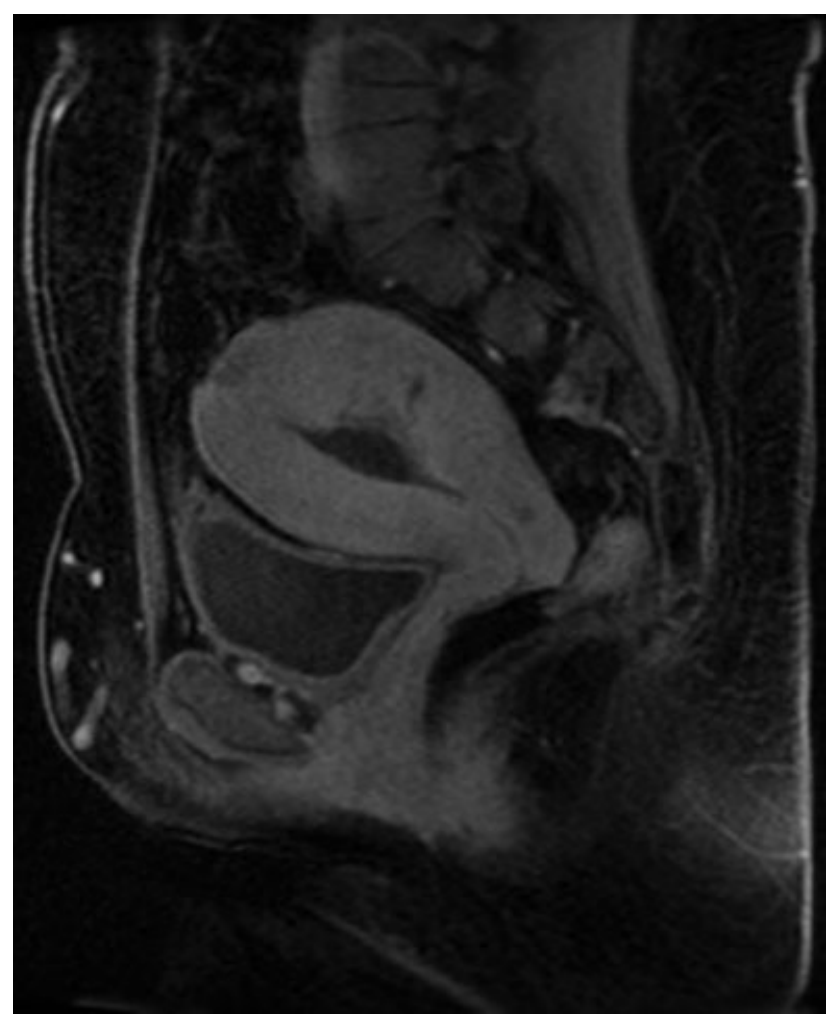

Fig. 7 Sagittal T1 postcontrast with fat saturation MR image revealing a complete treatment response status post uterine artery embolization and myomectomy, with marked reduction in size of the uterus. 


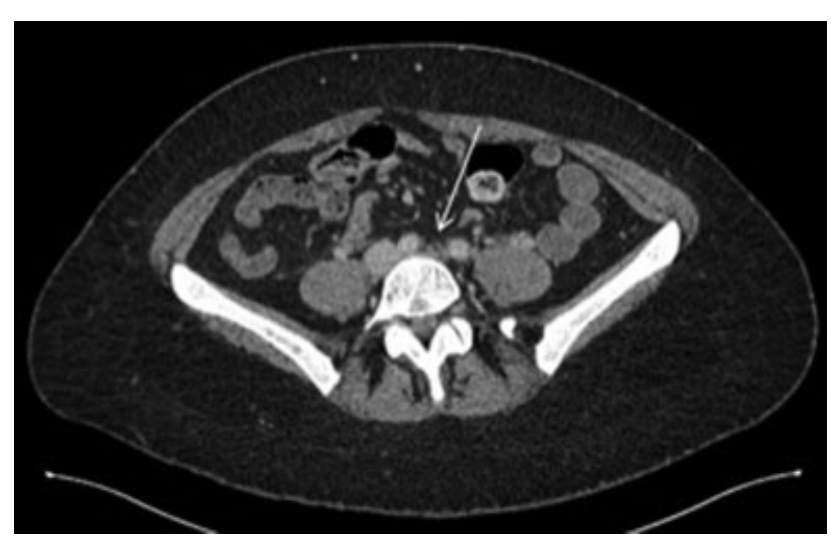

Fig. 8 Post-contrast axial CT image of the pelvis showing occlusion/ near occlusion of the left common iliac vein at its overlap with the right common iliac artery (arrow).

overlying right common iliac artery, with diminutive caliber of the remainder of the left common and external iliac veins suggesting chronic thrombosis (- Fig. 8).

\section{Procedure 2}

Her pelvic vein reconstruction was then scheduled and performed in a single session. Access was obtained via a left popliteal approach. Venography revealed the left common, femoral, external, and common iliac veins to be atretic and irregular, with an extensive pelvic collateral network (-Fig. 9a-c). Once crossed, serial prolonged iliofemoral venoplasty $(14 \mathrm{~mm})$ was performed, with effacement of multifocal high-grade stenoses. Intravascular ultrasound (IVUS) was then used to characterize the May-Thurner lesion and choose appropriate stents. A $14 \mathrm{~mm} \times 12 \mathrm{~cm}$ Life Star stent (Bard Peripheral Vascular, Tempe, AZ) was deployed in the left common and external iliac arteries, with an overlapping $12 \mathrm{~mm} \times 12 \mathrm{~cm}$ Life Star stent deployed distally extending into the common femoral vein. After postdilating the stent, follow-up IVUS revealed markedly improved luminal caliber, confirmed with a completion venogram showing brisk in-line flow centrally and no filling of collateral branches ( - Fig. 9d). Attention was then turned to retrieving the IVC filter, which was retrieved uneventfully. There was no evidence of caval thrombus (-Fig. 9e). After the procedure, $300 \mathrm{mg}$ of clopidogrel was administered, after which she was started on $75 \mathrm{mg}$ clopidogrel PO daily for 1 month in addition to her rivaroxaban.

At her follow-up visit in the hematology clinic, treatment recommendations included continuing the clopidogrel for 1 month and rivaroxaban for 3 months, at which time she should transition to life-long low-dose aspirin. It was also noted that prophylactic anticoagulation be considered in the future for high-risk scenarios.

At her 1-month follow-up visit in the IR clinic, the patient was doing well, back to her normal activities, and with subjective less left leg swelling. On physical examination, her calf circumference was not significantly changed, but her left thigh circumference was $4 \mathrm{~cm}$ smaller compared with her prestenting measurement.

\section{Discussion}

Uterine artery embolization is a ubiquitous procedure and a broadly recognized alternative to surgical interventions for symptomatic leiomyomata when uterine preservation is desired. Aside from postembolization syndrome (typically considered an expected feature of recovery), the most frequently described complications are temporary or permanent amenorrhea and lingering vaginal discharge. Less frequently described complications include fibroid expulsion (FE), protracted or refractory pain, infection, urinary retention, and access-related injuries. Other rare complications include death due to pulmonary embolism or sepsis, unintended embolization of leiomyosarcoma, uterine/buttock/labial necrosis, and vesicouterine fistulae. ${ }^{1}$

Transcervical leiomyoma expulsion is the process whereby necrotic fibroid material is passed into the endometrial cavity and through the cervix after UAE. Reported rates of transcervical FE vary in the literature from 3 to $50 \%$ but are most often quoted to be around 5 to $15 \%{ }^{2}$ Variability in reporting is likely related to the inconsistent nature of patient self-reporting and definitions of FE.

Two types of FE are described by Shlansky-Goldberg et al as "bulk" and "sloughing." Bulk expulsion entails passage of an entire fibroid or large fragments thereof, as our patient experienced. Sloughing occurs when the tumor liquefies over time and communicates with the endometrium, manifesting as a chronic vaginal discharge. ${ }^{3}$

Certain features predispose a patient to FE, including size and location of the tumor, with pedunculated submucosal, submucosal, and transmural lesions considered to be "high risk. ${ }^{3,4}$ Verma et al found that the larger the size of the fibroid and the more it interfaces with the endometrium on MRI, the more likely it is to be expelled following UAE. ${ }^{4}$ Expulsion may occur at once, or the fibroid may fragment and pass over time; associated symptoms include pain, bleeding, and fever.

While the optimal management of $\mathrm{FE}$ has not been definitively determined, high rates of nonoperative management of FE are noted in the literature, with 12 of 13 patients in the EMMY trial managed nonoperatively. ${ }^{2,3}$ MRI can help triage patients and determine when an additional intervention, such as transvaginal myomectomy, hysteroscopy, or even hysterectomy, may be indicated; nulliparity may predispose patients to failure of nonoperative management. ${ }^{3}$

When the patient presented with bulk expulsion in the setting of an open endocervical canal on MRI and the determination made that she should undergo myomectomy, perioperative VTE prophylaxis with caval filtration was warranted for her iliofemoral DVT. ${ }^{5}$ A retrievable suprarenal IVC filter was placed prior to her gynecologic surgery, but she was otherwise maintained on anticoagulation throughout her clinical course, as prompt and adequate anticoagulation is thought to help prevent PTS. ${ }^{6}$

The patient's extensive left lower extremity DVT was provoked in the setting of a massively enlarged, leiomyomatous uterus exerting further mass effect upon the already narrowed left common iliac vein from her May-Thurner lesion. While her DVT was considered chronic by the time her pelvic vein 

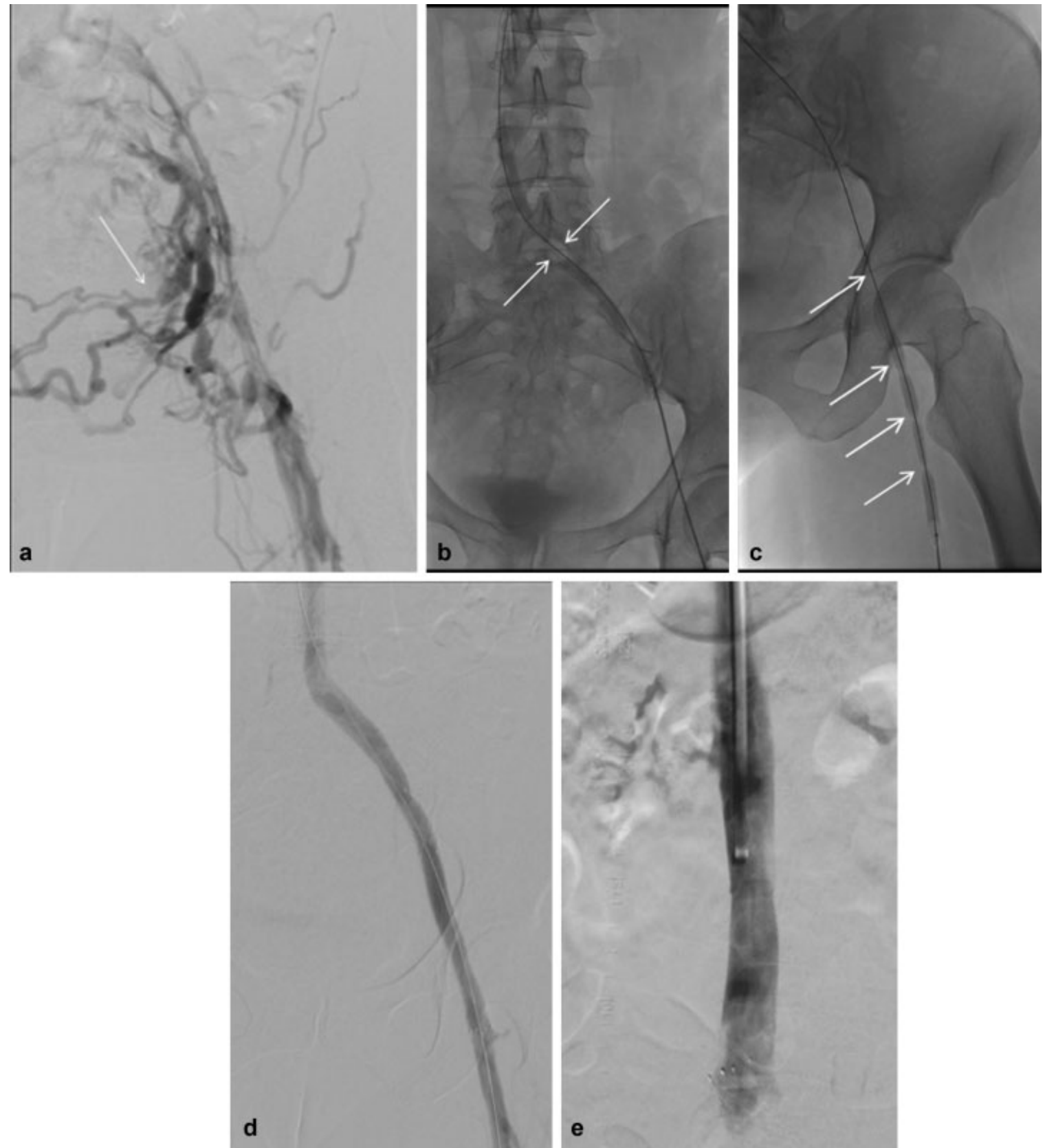

Fig. 9 (a-c) Digital subtraction angiographic images from pelvic venous reconstruction and inferior vena cava (IVC) filter retrieval. Images showed high-grade stenosis of the left common iliac vein due to external compression by the right common iliac artery (arrows), with long segment near occlusion of the left common iliac vein, left external iliac vein, left common femoral vein, and proximal left femoral vein (arrow), with heavy pelvic collateralization (arrow). (d) Overlapping 14- and 12-mm stents were placed from the left iliac vein through the proximal left common femoral vein, with markedly improved luminal caliber throughout the iliofemoral system, brisk in-line flow into the IVC, and no filling of collateral branches. (e) Completion venogram following filter retrieval via a right internal jugular vein (IJ) approach.

reconstruction was performed, a good technical result was obtained in a single session with mechanical thrombectomy and endovascular stenting to relieve the outflow obstruction. Her IVC filter was also retrieved at the time of this procedure. There is evidence of durable primary patency rates of stents placed for May-Thurner syndrome, even in the setting of chronic DVT. ${ }^{7}$ Studies show a trend of increasing PTS symptoms associated with delayed treatment, and that catheterdirected thrombolysis is effective for reducing PTS even in the setting of chronic, refractory DVT. ${ }^{7,8}$
In this case, a multidisciplinary approach facilitated timely, patient-centered care, and ultimately a positive clinical outcome. It also underscores the importance of a complete workup for DVT, which unmasked not only a May-Thurner lesion but also a symptomatic leiomyoma. While FE is an unusual complication following UAE, additional gynecologic and IR procedures were still required for comprehensive management in this case.

\section{Disclosure}

The authors report no relevant financial disclosure. 


\section{Fibroid Expulsion after Uterine Artery Embolization Ochmanek et al.}

\section{References}

1 Dariushnia SR, Nikolic B, Stokes LS, Spies JB; Society of Interventional Radiology Standards of Practice Committee. Quality improvement guidelines for uterine artery embolization for symptomatic leiomyomata. J Vasc Interv Radiol 2014;25(11):1737-1747

2 Hehenkamp WJK, Volkers NA, Donderwinkel PFJ, et al. Uterine artery embolization versus hysterectomy in the treatment of symptomatic uterine fibroids (EMMY trial): peri- and postprocedural results from a randomized controlled trial. Am J Obstet Gynecol 2005;193(05):1618-1629

3 Shlansky-Goldberg RD, Coryell L, Stavropoulos SW, et al. Outcomes following fibroid expulsion after uterine artery embolization. J Vasc Interv Radiol 2011;22(11):1586-1593

4 Verma SK, Bergin D, Gonsalves CF, Mitchell DG, Lev-Toaff AS, Parker L. Submucosal fibroids becoming endocavitary following uterine artery embolization: risk assessment by MRI. AJR Am J Roentgenol 2008;190(05):1220-1226
5 Caplin DM, Nikolic B, Kalva SP, Ganguli S, Saad WEA, Zuckerman DA; Society of Interventional Radiology Standards of Practice Committee. Quality improvement guidelines for the performance of inferior vena cava filter placement for the prevention of pulmonary embolism. J Vasc Interv Radiol 2011;22(11): 1499-1506

6 Ten Cate-Hoek AJ. Prevention and treatment of the post-thrombotic syndrome. Res Pract Thromb Haemost 2018;2(02):209-219

7 Craig P, Zuchowski A, Young S, Golzarian J, Rosenberg M. Results of endovascular management of May-Thurner syndrome in the acute, subacute, and chronic setting. J Vasc Interv Radiol 2017; 28(02):S113

8 Garcia M, Sterling K, Jaff M, et al. ACCESS PTS Study: accelerated thrombolysis for post-thrombotic syndrome using the acoustic pulse thrombolysis EkoSonic endovascular system: midterm results of a multicenter study. J Vasc Interv Radiol 2018;29(04): S151 